\title{
Rabdomiolisis inducida por el ejercicio
}

\author{
Mạ. P. Pérez Unanua, J. C. Roiz Fernández*, R. Diazaraque Marín** \\ Médico de familia. Centro de Salud Ciudad de los Periodistas. Madrid. \\ *Médico interno residente de tercer año de Medicina Familiar y Comunitaria. Centro de \\ Salud Ciudad de los Periodistas. Madrid. **Médico interno residente de Medicina Interna. \\ Hospital La Paz. Madrid
}

\section{RESUMEN}

La rabdomiolisis constituye un síndrome clínico y bioquímico resultado del daño muscular, necrosis del músculo esquelético y liberación del contenido celular al torrente circulatorio. Existe una gran he terogeneidad en la forma de presentación clínica, los pacientes suelen referir dolores musculares, debilidad y malestar general, pero las complicacio nes pueden ser severas: arritmias cardiacas y fracaso renal agudo. Se han descrito numerosas causas (enfermedades hereditarias del metabolis mo, traumatismos, ejercicio, fármacos, inmoviliza ción, trastornos metabólicos, enfermedades infec ciosas, etc.). Presentamos el caso de un varón de 38 años, bombero de profesión y gran deportista, que refería una historia de dolor muscular, calam bres y debilidad de dos días de evolución, tras la práctica de ejercicio.

Palabras clave: Rabdomiolisis. Ejercicio. Crea tina kinasa.

\section{Exercise-induced rhabdomyolysis}

\section{INTRODUCCIÓN}

La rabdomiolisis constituye un síndrome que, desencadenado de forma aguda o subaguda, conduce a la necrosis de las células del músculo esquelético. El síndrome clínico y bioquímico es el resultado del daño muscular y de la liberación del contenido celular al torrente sanguíneo. Presenta un amplio espectro de manifestaciones clínicas y analíticas, que dependen fundamentalmente de la severidad del daño muscular y del agente etiológico implicado, des-

\begin{abstract}
Rhabdomyolisis is defined as a clinical and bio chemical syndrome resulting from skeletal muscle injury that alters the integrity of the muscle cell membrane sufficiently to allow the release of the muscle cell contents into the plasma. There is a great heterogeneity in the clinical presentation of rhabdomyolysis. Patiens often have swollen and painful muscles. Cardiac arrest, and acute renal failure are the major complications. Numerous pre cipitating factors of rhabdomyolysis have been des cribed (hereditary enzyme defects, exercise, drugs, trauma, immobilization, metabolic acidosis, infec tious diseases,...). We present the case of a 38 year old male, fireman and sportman, who had a two days history of myalgias, tenderness and weakness after extreme exercise.
\end{abstract}

Key words: Rhabdomyolysis. Exercise. Creatinekinase. de moderados síntomas musculares hasta situaciones de riesgo vital que requieren una intervención urgente $^{1}$. Las manifestaciones clínicas de la rabdomiolisis se dividen en síntomas y signos musculares, sintomatología general y complicaciones asociadas. Los signos y síntomas musculares consisten en dolor, debilidad, calambres y contracturas, pueden ser generalizados o afectar a un solo grupo de músculos. Manifestaciones generales como fiebre, taquicardia, náuseas, vómitos, dolor abdominal y malestar general, disminución del nivel de 
consciencia, agitación, confusión y coma. Las complicaciones secundarias más importantes son las arritmias cardiacas, el síndrome compartimental y el fracaso renal agudo ${ }^{1}$. A continuación vamos a comentar el caso de un paciente con un cuadro de rabdomiolisis leve inducida por ejercicio, cuyo diagnóstico y seguimiento fue realizado desde la consulta de Atención Primaria.

\section{OBSERVACIÓN CLÍNICA}

Varón de 38 años que acude a la consulta del Centro de Salud por calambres, dolor y debilidad en ambos miembros inferiores de 48 horas de evolución. Entre sus antecedentes personales destaca el diagnóstico de esteatosis hepática dos años antes. Bombero de profesión, practica deporte con regularidad (atletismo y ciclismo). Los días anteriores al cuadro clínico había estado entrenando para una carrera ciclista de larga duración. En la exploración física se objetiva una auscultación cardiopulmonar normal, abdomen normal, complexión atlética e hipertrofia muscular con pérdida de fuerza 3/5 en ambos miembros inferiores, ROT presentes y tensión arterial de 120/85 $\mathrm{mmHg}$. En la consulta se realiza un Combur test ${ }^{\circledR}$ con resultado negativo. El paciente niega la ingesta de drogas, fármacos y sustancias anabolizantes. Ante la sospecha diagnóstica se solicita una analítica urgente: leucocitos: $7.500 / \mu 1$ (fórmula normal), hemoglobina $17 \mathrm{~g} / \mathrm{l}$, hematocrito 49\%, VCM $82 \mathrm{fl}$, plaquetas $245.000 / \mu 1$, glucosa $117 \mathrm{mg} / \mathrm{dl}$, creatinina 0,9 mg/dl, GOT 126 U/l, GPT 119 U/l, GGT 51 U/l, FA 107 U/l, LDH 460 U/l, CPK 2453 U/l (25-200), $\mathrm{Na} 142 \mathrm{mM} / 1$ y K 4,57mM/l. TSH $1,57 \mu \mathrm{U} / \mathrm{ml}$. Fue tramitada la incapacidad laboral del paciente, aconsejando reposo, hidratación abundante y nuevo control analítico a los 3 días.

El paciente permanece en reposo con mejoría clínica de los síntomas. Se realizó control analítico a los tres días: creatinina $1 \mathrm{mg} / \mathrm{dl}$, urea 35 $\mathrm{mg} / \mathrm{dl}$, CPK $965 \mathrm{U} / \mathrm{l}$, aldolasa 8,7 U/1 (0,0-7,5), GOT 73 U/l, GPT 113 U/l, GGT 55 U/l; a la semana: creatinina 1 , urea 42 , CPK $368 \mathrm{U} / 1$, GOT $37 \mathrm{U} / 1$, GPT $62 \mathrm{U} / 1$, GGT $50 \mathrm{U} / 1$, Na $145 \mathrm{mM} / 1$, $\mathrm{K} 5 \mathrm{mM} / 1$ y a los 15 días: glucosa $96 \mathrm{mg} / \mathrm{dl}$, creatinina $0.9 \mathrm{mg} / \mathrm{dl}$, urea $34 \mathrm{mg} / \mathrm{dl}$, colesterol 318 $\mathrm{mg} / \mathrm{dl}$, GOT $41 \mathrm{U} / 1$, GPT $54 \mathrm{U} / 1$, GGT $50 \mathrm{U} / 1$, CPK 620 U/1, Na 142 mM/l y K 4,31 mM/l. El paciente se reincorporó a su actividad laboral, aconsejando disminuir el ejercicio físico que, hasta entonces, había practicado intensa y regularmente. Ante la persistencia de niveles elevados de CPK: $927 \mathrm{U} / 1$ al mes siguiente, $535 \mathrm{U} / 1$ a los dos meses, se solicitó la realización de un electromiograma que reflejaba cambios miopáticos difusos en MMSS y MMII de grado leve moderado, sin da- tos de destrucción muscular aguda extensa en ese momento, es decir, no hay signos de proceso activo sino que se trata de cambios crónicos, es posible que se trate de cambios secuelares tras un episodio agudo de rabdomiolisis. Así mismo fue realizada una biopsia muscular (cuatro meses después del episodio agudo): el estudio histológico de la pieza muestra una arquitectura conservada sin fibrosis o componente inflamatorio. No presenta signos de vasculitis. Existe una variabilidad en el diámetro de las fibras dentro de la normalidad. No signos de necrosis ni regeneración de las fibras. No se observan depósitos de glucógeno ni de lípidos. El estudio con microscopía electrónica no revela anomalías estructurales de las mitocondrias, algunas de las cuales presentan un tamaño patológico. Se realizaron en otro hospital estudios bioquímicos de función mitocondrial con resultado en rango de normalidad. Diagnóstico final anatomopatológico: músculo estriado con mínimas alteraciones.

Durante estos meses se ha realizado un seguimiento analítico del paciente en el que hemos comprobado una práctica normalización de las transaminasas, persistiendo una leve elevación de la GGT compatible con el diagnóstico previo de esteatosis hepática y niveles ligeramente elevados de CPK, probablemente consecuencia de la enorme masa muscular del paciente. Diagnóstico final: rabdomiolisis inducida por ejercicio.

\section{DISCUSIÓN}

Los criterios diagnósticos de rabdomiolisis no están establecidos de forma estricta debido a la diversidad clínica del cuadro ${ }^{2}$. En la mayoría de los casos de basan en la presencia de a) evento desencadenante de destrucción muscular; b) incremento de los niveles de enzimas musculoesqueléticas; y c) incremento en los niveles de mioglobina sérica y mioglobinuria.

En cuanto a su fisiopatología, podemos decir que la rabdomiolisis es el resultado de daño muscular directo o bien de un disbalance energético en el músculo que interfiere en la homeostasis del calcio así como en el correcto funcionamiento de la bomba $\mathrm{Na} / \mathrm{K}$ ATPasa que son de vital importancia para mantener la integridad de la membrana celular. De esta forma se liberan al torrente sanguíneo sustancias nocivas para la salud ${ }^{3}$.

La rabdomiolisis presenta dos problemas importantes: a) rabdomiolisis severa con complicaciones secundarias de riesgo vital; y b) rabdomiolisis levemoderada de repetición, que precisa estudio para determinar posibles defectos metabólicos subyacentes.

Hay varias clasificaciones de rabdomiolisis referidas a su etiología. Podemos dividir las causas de 
rabdomiolisis en traumáticas y no traumáticas, endógena y exógena o formas hereditarias y adquiridas. Preferimos esta última clasificación por resultar más sencilla. En cuanto a las formas hereditarias tenemos las enfermedades metabólicas del glucógeno y de los lípidos, la hipertermia maligna e idiopáticas. En las formas no hereditarias el paciente ha sido sometido a circunstancias extremas: ejercicio, traumatismos, isquemia muscular, enfermedades infecciosas ${ }^{4}$, drogas y toxinas 5 (Tabla I).

\section{Tabla I}

\begin{tabular}{|c|c|}
\hline \multicolumn{2}{|c|}{ CAUSAS DE RABDOMIOLISIS } \\
\hline \multicolumn{2}{|r|}{ HEREDITARIAS } \\
\hline \multicolumn{2}{|r|}{ Déficits enzimáticos } \\
\hline $\begin{array}{l}\text { 1. Alteraciones en el } \\
\text { metabolismo del } \\
\text { glucógeno: }\end{array}$ & $\begin{array}{l}\text { Miofosforilasa (enf. De McArdle) } \\
\text { Fosforilasa kinasa } \\
\text { Fosfofructokinasa (enf. De Tarui) } \\
\text { Fosfoglicerato kinasa } \\
\text { Fosfoglicerato mutasa } \\
\text { Lactato deshidrogenasa }\end{array}$ \\
\hline $\begin{array}{l}\text { 2. Alteraciones del } \\
\text { metabolismo } \\
\text { de los lípidos }\end{array}$ & $\begin{array}{l}\text { Déficit de carnitin palmitoil transferasa I y II } \\
\text { Déficit de carnitina }\end{array}$ \\
\hline $\begin{array}{l}\text { 3. Otras: rabdomiolis } \\
\text { deaminasa, hipert } \\
\text { maligno. }\end{array}$ & $\begin{array}{l}\text { is idiopática, déficit de mioadenilato } \\
\text { ermia maligna, síndrome neuroléptico }\end{array}$ \\
\hline
\end{tabular}

ADQUIRIDAS

1. Tóxicos: alcohol, opiaceos, cocaína, anfetaminas.

2. Fármacos: fenciclidina, neurolépticos, barbitúricos, teofilinas, antihistamínicos, fibratos, estatinas, ácido epsilonaminocaproico, antibióticos, azatioprina, anfotericina B.

3. Toxinas: succinilcolina, toxina tétanos, tifus, estafilococo, venenos de serpiente, monóxido de carbono, tolueno

4. Ejercicio muscular excesivo: deporte, estatus epiléptico, asma, distonías

5. Daño muscular directo: traumatismo, quemadura, inmovilización

6. Isquemia: compresión vascular, infarto muscular

7. Trastornos metabólicos: cetoacidosis diabética, coma hiperosmolar, hipotiroidismo, hipofosfatemia, hiponatremia, hipokalemia

8. Enfermedades infecciosas: bacterianas (legionella, estreptococo, salmonella) o víricas (influenza, varicela zóster y $\mathrm{VIH})$

9. Miopatías: polimiositis y dermatomiositis.

10. Otras: síndrome anticolinérgico, retirada de L-Dopa

Modificada de cita 2.
Ejercicio y rabdomiolisis pueden estar relacionados por diferentes motivos. El ejercicio es el principal factor precipitante de rabdomiolisis en el caso de las enfermedades metabólicas. El cuadro clínico aparece en individuos jóvenes, que presentan episodios recurrentes de rabdomiolisis y mantienen una elevación persistente de la CPK. En estos pacientes está indicada la realización de biopsia muscular ${ }^{6}$.

El entrenamiento disminuye el riesgo de desarrollar rabdomiolisis, pero en ausencia de aclimatación al calor, sudoración profusa, falta de reposición hidroelectrolítica adecuada y elevada temperatura ambiental, puede afectar incluso a deportistas entrenados como sería nuestro $\mathrm{caso}^{7}$. Cualquier persona sana sometida a un ejercicio extremo y extenuante puede presentar un episodio de rabdomiolisis ${ }^{8,9}$. En una serie de 35 casos de rabdomiolisis inducida por ejercicio no se describió fallo renal en ninguno de los $\operatorname{casos}^{10}$, lo que hace pensar que es necesario la presencia añadida de cofactores nefrotóxicos (hipovolemia marcada y/o acidosis/aciduria, isquemia renal) para desencadenar el fracaso renal ${ }^{11}$.

El diagnóstico se basa como hemos visto antes en síntomas clínicos musculares, la presencia de un factor desencadenante y hallazgos de laboratorio. Los datos de laboratorio más frecuentes son la elevación de la mioglobina, CPK, y otros enzimas musculares. La elevación de la mioglobina (normal: $3-80 \mu \mathrm{g} / \mathrm{l}$ ) precede a la de la CPK, pero se normaliza a las 6 horas del cese del daño muscular, con lo que resulta útil en el diagnóstico precoz, pero valores normales no descartan el diagnóstico. Tiene bajo peso molecular por lo que se filtra rápidamente por el glomérulo renal y tiñe la orina cuando su concentración excede de $1 \mathrm{~g} / 1$. La mioglobinuria sólo se detecta en fases iniciales del cuadro, en nuestro caso la tira reactiva de orina fue negativa ${ }^{12,13}$. La alteración más sugestiva es la elevación de la CPK (al menos x 5 veces su valor, normal: $150 \mathrm{UI} / \mathrm{l})$. Se eleva a las 2-12 horas del daño muscular con un pico en 1-3 días para descender a los 3-5 días. Una elevación moderada y persistente de la CPK se presenta en miopatías, hipotiroidismo, enfermedades metabólicas, etc. La anhidrasa carbónica III es el marcador más específico de daño muscular, aunque la mayoría de las veces no es posible su determinación. También se puede detectar una elevación de las transaminasas (GOT/ASAT, GPT/ALAT), aldolasa, LDH, creatinina, urea, ácido úrico. Al alterarse la permeabilidad de la membrana celular podemos encontrar hiperpotasemia, hipocalcemia e hiperfosfatemia, con el consiguiente riesgo de arritmias cardiacas, calcificaciones tisulares, etc.

Una vez confirmado el diagnóstico y estimado el riesgo de complicaciones, y sólo en el caso de que la patogenia no esté clara o rabdomiolisis recurren- 
te se investiga acerca de las enfermedades genéticas del metabolismo muscular.

El electromiograma puede resultar de utilidad en fase aguda. La determinación en sangre de la actividad de las enzimas del metabolismo del glucógeno y lípidos requiere la intervención del especialista. La biopsia muscular es la prueba fundamental, se observa necrosis de las células del músculo y permite el estudio mediante técnicas de histoquímica, enzimáticas, bioquímica, cromatografía, electroforesis e inmunológicas de los distintos déficits estructurales y enzimáticos del músculo.

Finalmente podemos decir que la rabdomiolisis es un cuadro clínico poco frecuente pero que nos podemos encontrar en nuestra práctica clínica habitual. Los síntomas pueden variar desde leve mialgia a debilidad muscular severa e implicar situaciones de riesgo vital, con lo que hay que actuar con precaución. Existe una gran heterogeneidad en cuanto a los factores etiológicos. El tratamiento siempre debe ir orientado en primer lugar a la supresión del agente causal, si es que se conoce, soporte vital en el caso de fracaso renal agudo y arritmias, hidratación alcalina adecuada para favorecer la eliminación de los productos tóxicos, vigilar la función renal y reposo por parte del paciente.

\section{AGRADECIMIENTOS}

A la doctora $\mathrm{M}^{\mathrm{a}}$ Isabel García Lázaro médico de familia del Centro de Salud Ciudad de los Periodistas, a la doctora Carmen Fernández Capitán, médico internista del Servicio de Medicina Interna del Hospital La Paz de Madrid y a los Servicios de Anatomía Patológica del Hospital La Paz y Doce de Octubre de Madrid.

\author{
CORRESPONDENCIA: \\ $M^{a}$. Paz Pérez Unanua \\ Plaza Peña Horcajo ${ }^{\circ} 11$ - portal B, $5^{\circ} \mathrm{B}$ \\ 28035 Madrid \\ Tel.: 91-7310175 \\ e-mail: mpperezunanua@hotmail.com
}

\section{Bibliografía}

1. Visweraran P, Guntupalli J. Rhabdomyolysis. Crit Care Clin 1999; 15: 415-28.

2. Poels PJE, Gabreëls FJM. Rhabdomyolysis: a review of literature. Clin Neurol Neurosurg 1993; 95: 175-92.

3. Bollaert PE, Frisoni A. Epidemiology, mechanism and clinical features of rhabdomyolysis. Minerva Anestesiol 1999; 65: 245-9.

4. Upinder S, Scheld WM. Infectious etiologies of rhabdomyolysis: three case report and review. Clin Infectious Diseases 1996; 22: 642-9.

5. Jermain DM, Lynn Crismon M. Psychotropic drug-related rhabdomyolisis. Ann Pharmacother 1992; 26: 948-54.

6. Olerud JE, Homer LD, Carroll HW. Incidence of acute exertional rhabdomyolysis. Arch Intern Med 1976; 136: 692-7.

7. Hammer R. When exercise goes agry: exertional rhabdomyolysis. South Med J 1997; 90: 548-51.
8. Sharma N, Winpenny H, Heyman T. Exercise-induced rhabdomyolysis: even the fit may suffer. Int J Clin Pract 1999; 53: 476-7.

9. Pintor Holguin E, Pardo Moreno J, Saez Noguero F, Ruiz Yagüe M, Fernandez Cruz A. Rabdomiolisis masiva después de la primera sesion de ejercicios de musculación. An Med Interna 1997; 14: 158-9.

10. Sinert R, Kohl L, Rainone T, Scalea T. Exercise-induced Rhabdomyolysis. Ann Emerg Med 1994; 23: 1301-6.

11. Ward MM. Factors predictive of acute renal failure in rhabdomyolysis. Arch Intern Med 1988; 148: 1563-7.

12. Holt S, Moore K. Pathogenesis of renal failure in rhabdomyolysis: the role of myoglobin. Exp Nephrol 2000; 8: 72-6.

13. O’Donnell J, Gleeson AP. Exercise induced rhabdomyolysis. Eur J Emerg Med 1998; 5: 325-6. 